\title{
Entwicklung der Rechtschreibkompetenz im zweiten und dritten Schuljahr: Eine latente Transitionsanalyse zur Überprüfung theoretischer Annahmen
}

\author{
Jessica Jaeuthe $\mathbb{D} \cdot$ Jennifer Lambrecht $\cdot$ Stefanie Bosse $\cdot$ Katja Bogda \\ Nadine Spörer
}

Online publiziert: 4. August 2020

(C) Der/die Autor(en) 2020

Zusammenfassung Im deutschsprachigen Raum existiert eine Vielzahl von Modellen zur Entwicklung der Rechtschreibkompetenz von Grundschulkindern. Es zeigen sich starke Übereinstimmungen in der Vorstellung von aufeinanderfolgenden Kompetenzniveaus, wobei in allen Modellen drei Niveaus auftreten: 1) noch nicht lautgetreue Schreibungen, 2) lautgetreue Schreibungen und 3) orthographisch korrekte Schreibungen. Die Kriterien, auf Basis derer ein Kind dem jeweiligen Niveau zugeordnet wird, bleiben jedoch vage. Ebenso fehlt eine umfassende empirische Überprüfung der Modelle. Die vorliegende Längsschnittstudie untersuchte zu drei Messzeitpunkten (Anfang Klasse 2, Ende Klasse 2, Ende Klasse 3) die Schreibungen von $N=697$ Grundschulkinder mit standardisierten Rechtschreibtests. Mittels latenter Transitionsanalyse wurden drei Profile identifiziert: 1) Überwiegend nicht lautgetreu 2) Überwiegend lautgetreu und 3) Überwiegend korrekt. Auch die durchlaufenen Pfade und Übergangswahrscheinlichkeiten stützen die angenommene hierarchische Struktur der Modelle.

\footnotetext{
J. Jaeuthe $(\bowtie) \cdot$ Dr. J. Lambrecht · Dr. S. Bosse $\cdot$ K. Bogda · Prof. Dr. N. Spörer

Professur für Psychologische Grundschulpädagogik, Universität Potsdam, Karl-Liebknecht-Str.

24-25, Haus 24, 14476 Potsdam, Deutschland

E-Mail: jaeuthe@uni-potsdam.de

Dr. J. Lambrecht

E-Mail: jennifer.lambrecht@uni-potsdam.de

Dr. S. Bosse

E-Mail: stefanie.bosse@uni-potsdam.de

K. Bogda

E-Mail: bogda@uni-potsdam.de

Prof. Dr. N. Spörer

E-Mail: nadine.spoerer@uni-potsdam.de
} 
Schlüsselwörter Rechtschreibkompetenz · Latente Transitionsanalyse ·

Längsschnittstudie · Grundschule

\title{
Development of spelling competence in second and third grade: A latent transition analysis to verify theoretical assumptions
}

\begin{abstract}
Related to the German language, there exists a variety of theoretical models to describe the development of orthographical competence of primary school students. When comparing these models, similarities were identified regarding the concept of consecutive levels of competence. In each of the models three levels were hypothesized: 1) no phonemic spelling yet, 2) phonemic spelling, and 3) orthographically correct spelling. However, the criteria on which basis a child is assigned to a respective level remain vague. Likewise, empirical validation of the models is missing. In the present study, spelling competency of $N=697$ primary school students was assessed using standardized spelling tests at three measurement time points (begin of grade 2, end of grade 2, end of grade 3). By applying latent transition analysis, three profiles of development were identified: 1) predominantly no phonemic, 2) predominantly phonemic and 3) predominantly orthographical correct. In accordance to the theoretical assumptions, the traversed paths and probabilities of transitions supported the hierarchical structure of the levels of competence.
\end{abstract}

Keywords Latent transition analysis · Longitudinal study · Primary school · Spelling

\section{Einleitung}

Rechtschreibkompetenz wird in unserer Gesellschaft als eine Schlüsselqualifikation gesehen. Ziel der Schule ist es daher, dass alle Schülerinnen und Schüler Rechtschreibkompetenzen erwerben. Die Entwicklung der Rechtschreibkompetenz wurde bereits in zahlreichen theoretischen Modellen dargestellt. Die Modelle weisen eine Reihe von Gemeinsamkeiten und Unterschieden auf, wobei ihnen die Annahme einer hierarchischen Struktur der Entwicklung der Rechtschreibkompetenz gemeinsam ist. Zwar existieren verschiedene Studien zur Entwicklung der Rechtschreibkompetenz, eine direkte Überprüfung der hierarchischen Struktur der Modelle blieb bislang jedoch aus. Diesem Desiderat widmet sich der vorliegende Beitrag, indem die Entwicklung von Grundschulkindern über zwei Grundschuljahre hinweg längsschnittlich untersucht wurde.

\section{Theoretischer Hintergrund}

Rechtschreibkompetenz besteht aus verschiedenen Aspekten. Hierzu gehört, dass Schülerinnen und Schüler die Zeichensetzung beachten, über Fehlersensibilität verfügen, eigene Schreibungen korrigieren und insbesondere Rechtschreibstrategien anwenden (vgl. KMK 2005). Rechtschreibstrategien umfassen sowohl die Fähig- 


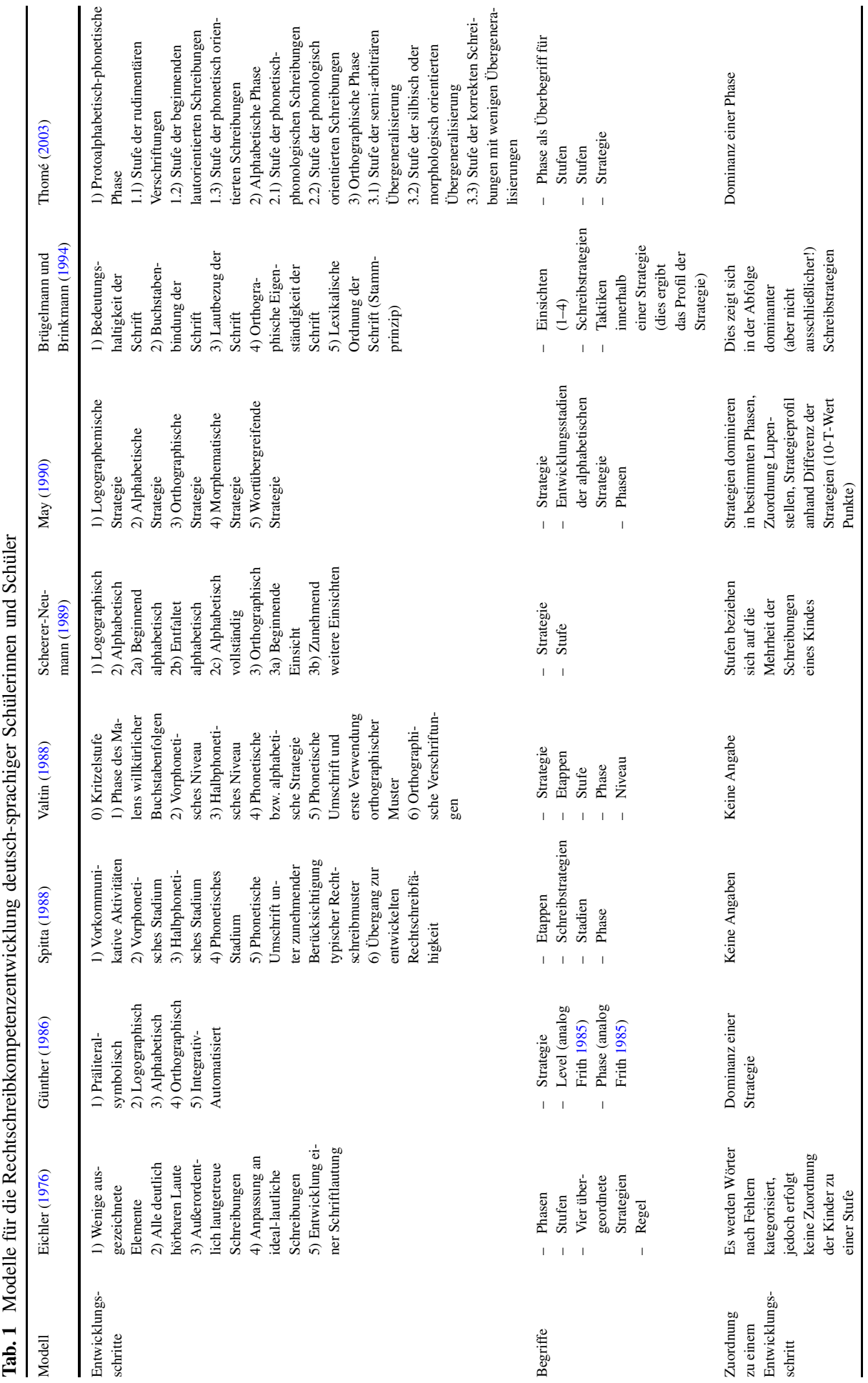


keit, Wörter zu schreiben, wie sie gesprochen werden (lautgetreues Schreiben), als auch das Berücksichtigen von orthographischen und morphematischen Regelungen (vgl. KMK 2005). Insbesondere diese Verwendung von Rechtschreibstrategien wird als zentral für die Entwicklung der Rechtschreibkompetenz betrachtet. Die Entwicklung der Rechtschreibkompetenz wird theoretisch als ein hierarchisch aufgebautes Kompetenzniveaumodell beschrieben.

Orthographie ist sprachgebunden, daher bestehen große Unterschiede für Modelle der Rechtschreibkompetenzentwicklung zwischen den Sprachen. Aus diesem Grund sollen nachfolgend die Modelle für die Entwicklung der deutschen Orthographie dargestellt werden. Für jedes der Modelle werden die angenommenen Entwicklungsschritte benannt, wobei die von den Autoren gewählten Begriffe berücksichtigt werden. Außerdem wird für jedes Modell geprüft, inwiefern Kriterien benannt werden, nach denen die Kinder einem Entwicklungsschritt zugeordnet werden können, da dies für die Überprüfung der hierarchischen Struktur relevant ist. Tab. 1 zeigt einen Überblick über die verschiedenen Modelle. Hierbei wurden nur solche Modelle in die Übersicht aufgenommen, die explizit eine Abfolge von verschiedenen Fähigkeiten definieren.

Als erstes Modell für die Entwicklung der deutschen Orthographie kann das Modell von Eichler aus dem Jahr 1976 angesehen werden. Hierbei beschreibt er die Entwicklung in drei Phasen: Er vermutet, dass sich Schülerinnen und Schüler in der ersten Phase bei der Verschriftlichung von Wörtern ausschließlich an ihrer Sprache orientieren, wobei zunächst ausschließlich besonders betonte oder gut hörbare Laute verschriftet werden. Es folgt ein konsequenter Ausbau der akustisch-auditiven Schreibweise, sodass sich die zweite Phase dadurch auszeichnet, dass es seltener zu Auslassungen von Graphemen innerhalb der Wörter kommt. Die dritte Phase der sogenannten Kinderschrift soll durch die Anpassung der Schreibungen an die Erwachsenenschreibung gekennzeichnet sein, wobei dies von Eichler (1976) mit dem dominanter werdenden Lesen begründet wird. In späteren Werken spricht Eichler (1992) von fünf Stufen. Auf der niedrigsten Stufe werden nur wenige Zeichen verschriftet, welche von den Kindern besondere Aufmerksamkeit erlangt haben. Auf der nächsten Stufe schreiben Schülerinnen und Schüler deutlich hörbare Laute. Darauf folgen laut Eichler (1992) die sogenannte außerordentlich lautgetreue Schreibung sowie die ideal-lautliche Schreibung. Auf der letzten Stufe entwickeln die Kinder eine Schriftlautung. Sowohl in früheren, als auch in späteren Werken mangelt es an Kriterien, nach denen Schülerinnen und Schüler einer Entwicklungsphase zugeordnet werden können.

Die Modelle von Spitta (1988) und Valtin (1988) zeigen große Ähnlichkeiten zu dem Modell von Eichler (1976). Sie unterscheiden sich jedoch dadurch, dass der Beginn der Entwicklung bereits in vorkommunikativen Aktivitäten (Spitta 1988) bzw. in einer Kritzelstufe (Valtin 1988) gesehen wird. Die weiteren Phasen unterscheiden sich vor allem in den gewählten Begriffen (siehe Tab. 1), jedoch weniger hinsichtlich der Inhalte. Allerdings kritisiert Valtin (1988; bzw. Valtin und Sasse 2018), dass sich die Modelle nur auf die Schreibung einzelner Wörter beziehen. Darüber hinaus sei auch wichtig, dass die Schülerinnen und Schüler über ein Wortkonzept verfügen. Analog zu Eichler (1976) werden keine Kriterien beschrieben, wie Schülerinnen und Schüler einer Stufe zugeordnet werden können. 
Das Modell von Günther (1986) basiert auf dem Model von Frith (1985), welches für die Entwicklung der englischen Orthographie entwickelt wurde. Hierbei werden fünf Phasen beschrieben: die präliteral-symbolische Phase, die logographische Phase, die alphabetische Phase, die orthographische Phase und die integrativ-automatisierte Phase. Analog zu Spitta (1988) und Valtin (1988) geht Günther (1986) davon aus, dass die Entwicklung der Orthographie bereits vor der Kenntnis der Buchstaben beginnt, indem die Kinder Zeichen produzieren. Als nächsten Entwicklungsschritt definiert er, dass (Vorschul-)Kinder ganze Wörter aus dem Gedächtnis schreiben können, indem sie diese wie ein Bild bzw. Logo gespeichert haben. Dieses Vorgehen wird als logographische Strategie bezeichnet. Parallel zur Aneignung von Buchstabenkenntnissen wird angenommen, dass Schülerinnen und Schüler beginnen so zu schreiben, wie sie sprechen (alphabetische Strategie), bevor sie allmählich auch orthographische Regeln beachten (orthographische Strategie). Jeweils eine Strategie soll in einer bestimmten Phase dominant zu beobachten sein, wobei die Übergänge zwischen den Phasen fließend sein können.

Scheerer-Neumann $(1989,2015)$ bezieht ihr Modell ebenfalls auf das Modell von Frith (1985). So existieren bei ihr die logographische, alphabetische und orthographische Stufe, wobei sowohl die alphabetische Stufe, als auch die orthographische Stufe differenzierter dargestellt werden. Sie geht darüber hinaus davon aus, dass Schülerinnen und Schüler zunehmend in der Lage sind, sich Schreibungen einzuprägen und automatisiert abzurufen, anstelle diese mühevoll zu konstruieren und beschreibt ihr Modell entsprechend als zwei-Wege-Modell. Der indirekte Weg beschreibt dabei die Entwicklung der Rechtschreibkompetenz entlang der verschiedenen Stufen, wohingegen auf dem direkten Weg Wörter aus dem orthographischen Lexikon direkt abgerufen werden können (z. B. Lernwörter). Die Stufen beziehen sich somit nur auf die Schreibung unbekannter Wörter. Die Schreibung eines Wortes ist demnach auch von der Sachstruktur des Wortes abhängig (vgl. Schründer-Lenzen 2013; Schneider 2017). Sehr leichte oder häufig vorkommende Wörter können von den Schülerinnen und Schülern durch den direkten Abruf früher richtig geschrieben werden, als schwierige oder seltener vorkommende Wörter. Werden den Schülerinnen oder Schülern hingegen unbekannte oder schwierige Wörter vorgegeben, so sollen sich nach Scheerer-Neumann (1989, 2015) qualitative Unterschiede zwischen den Schreibungen zeigen, da sich die Schülerinnen und Schüler auf unterschiedlichen Stufen befinden, wobei die Übergänge fließend sein können. Die Schreibung, die sodann von einem Kind mehrheitlich genutzt wird, bildet die Grundlage für die Zuordnung eines Kindes zu einer Stufe.

Ein weiteres Modell, welches zu einem ähnlichen Zeitpunkt entstand, stammt von May (1990, 2012). Das Modell von May (1990) zeigt große Ähnlichkeiten zu dem Modell von Scheerer-Neumann (1989, 2015). May (1990, 2012) beschreibt die Entwicklung als Abfolge von fünf Strategien: der logographischen Strategie, der alphabetischen Strategie, der orthographischen Strategie, der morphematischen Strategie und der wortübergreifenden Strategie. Die Unterschiede bestehen vor allem in der Trennung der orthographischen und morphematischen Strategie und der Hinzunahme einer wortübergreifenden Strategie. Mittels der Hamburger Schreibprobe (May 2012), ein standardisierter Rechtschreibtest für Schülerinnen und Schüler der ersten bis zehnten Klasse, wird ermittelt, wie stark jede Strategie ausgeprägt ist. 
Hierzu wird basierend auf der Schreibung sogenannter Lupenstellen ein altersnormierter T-Wert für jede Strategie ermittelt. Über den Vergleich der Ausprägungen wird schließlich der Entwicklungsstand bestimmt, wobei angenommen wird, dass Schülerinnen und Schüler zunächst über ein Profil mit alphabetischer Dominanz verfügen, dann mit alphabetisch-orthographischer Dominanz und abschließend über ein ausgeglichenes Profil ${ }^{1}$. Fraglich ist jedoch, inwiefern ein ausgeglichenes Profil ein angemessener Indikator für die höchste Entwicklungsstufe ist, weil auch jene Schülerinnen und Schüler, die keine Lupenstelle korrekt verschriftlichen, über ein ausgeglichenes Profil verfügen.

Brügelmann und Brinkmann (1994) bauen auf die bereits vorhandenen Modelle auf. So finden sich vor allem Ähnlichkeiten zu Valtin (1988), indem analog zunächst die Bedeutungshaltigkeit der Schrift (Phase 1) und die Buchstabenbindung der Schrift (Phase 2) an den Anfang gestellt werden, bevor Schülerinnen und Schüler den Lautbezug der Schrift erkennen (Phase 3). Daran schließen sich die Phase 4, in der die orthographische Eigenständigkeit der Schrift erkannt wird, sowie die Phase 5 an, in welcher die lexikalische Ordnung der Schrift im Sinne des Stammprinzips erfolgt. Brügelmann und Brinkmann (1994) benennen damit ähnlich wie May (1990, 2012) das Stammprinzip (bei May Morphematisches Prinzip) als eigenständigen Entwicklungsschritt. Die Zuordnung von Schülerinnen und Schüler zu einer Stufe erfolgt durch die Bestimmung der dominanten Strategie, wobei nicht genau definiert wird, was unter dominant verstanden wird.

Thomé (2003) beschreibt die Entwicklung von Rechtschreibkompetenz ausgehend von der Verwendung von Basisgraphemen und Orthographemen. Hierfür wurden Phonem-Graphem-Relationen aus Texten analysiert, um die häufigste Schreibung eines Phonems zu identifizieren. Als Basisgraphemen wird dabei die häufigste Schreibung eines Phonems benannt. So wurde in einer Auszählung von 10.000 Phonem-Graphem-Relationen in deutschen Texten deutlich, dass z. B. /i:/ in über $83 \%$ der Fälle als <ie> geschrieben wurde (Thomé 2003). Deutlich seltener tritt die Schreibung z. B. als <ih> auf, sodass diese Schreibung als Orthographem bezeichnet wird. Insgesamt vermutet Thomé (2003) drei Phasen, welche wiederum jeweils in zwei bzw. drei Stufen unterteilt werden, die von Schülerinnen und Schülern durchlaufen werden sollen. Auch in seinem Modell gibt es eine Phase, in der die Kinder zunächst schriftähnliche Kritzeltexte verfassen. In der anschließenden alphabetischen Phase orientieren sich die Kinder an ihrer Aussprache und können dann Wörter, welche ausschließlich aus Basisgraphemen bestehen, richtig schreiben. Erst in der letzten Phase werden über die Basisgrapheme hinaus Orthographeme verwendet. Auf eine logographische Phase möchte Thomé (2003) bewusst verzichten. In der Entwicklung soll jeweils eine Phase dominant auftreten. Genaue Kriterien, wie Kinder einer Phase oder Stufe zugeordnet werden können, bleiben offen.

\footnotetext{
1 Mit einem ausgeglichenen Profil ist gemeint, dass die T-Werte für die einzelnen Strategien sich nur um weniger als 10 T-Wert Punkte unterscheiden. Dies soll vor allem dann der Fall sein, wenn die Schülerinnen und Schüler in allen Strategien möglichst viele Lupenstellen korrekt geschrieben haben. Jedoch müsste auch dann von einem ausgeglichenen Profil gesprochen werden, wenn keine der Lupenstellen richtig verschriftet wurde, da auch dann sich die T-Werte der einzelnen Strategien nicht unterscheiden.
} 
Die bisher beschriebenen Kompetenzmodelle ähneln sich insofern, dass all diese Modelle die Entwicklung der Rechtschreibkompetenz als Abfolge von aufeinander aufbauenden Kompetenzen beschreiben. Darüber hinaus existiert auch das Rechtschreibkompetenzmodell von Blatt und Jarsinski (2009). Die Autoren beschreiben hierbei fünf Teilfähigkeiten, welche sich auf unterschiedliche graphematische Strukturen beziehen, wobei die Silbenstruktur der Wörter von den Schülerinnen und Schülern berücksichtigt werden muss. Die Autoren definieren dabei folgende Teilfähigkeiten: Herstellen von Bezügen zwischen Schrift und Lautstruktur, Flexionsmorpheme kennen und anwenden, Markierungen in offenen Silben setzen, Wortbildungsmorpheme kennen sowie Syntaxstrukturen kennen (Blatt und Jarsinski 2009). Inwiefern es sich bei den Prinzipien um aufeinander aufbauende Teilfähigkeiten handelt, wird nicht näher erläutert. Darüber hinaus werden keine Kriterien angegeben, ab wann eine Teilfähigkeit von den Schülerinnen und Schülern beherrscht wird.

Ein weiteres Modell wurde von Löffler und Meyer-Schepers (2009) für den Test DoSe in IGLU 2001 entwickelt. In diesem Modell wird Rechtschreibkompetenz als zweidimensionales Konstrukt verstanden. Es wird zwischen einer lautanalytische und einer grammatische Dimension unterschieden, welche jeweils auf zwei Ebenen von den Schülerinnen und Schülern beherrscht werden sollen: eine elementare und eine erweiterte Ebene. Die Schreibungen der Schülerinnen und Schüler werden hinsichtlich der Einzelfehler analysiert, wobei jeder Fehlschreibung einer Dimension und einer Ebene zugeordnet wird. Insgesamt entsteht so eine Diagnose für jedes Kind, wobei betrachtet wird, inwiefern die Fähigkeiten des Kindes vom Durchschnitt der Leistungen der jeweiligen Klassenstufe liegen.

Beim Vergleich der Modelle wird deutlich, dass von den Autoren nicht nur unterschiedlich viele Entwicklungsschritte angegeben werden, sondern auch unterschiedliche Begriffe verwendet werden. So finden sich die Begriffe Phasen, Stufen, Strategien, Level, Regeln, Einsichten, Taktiken, Kategorien, Etappen, Niveaus, Stadien, Prinzipien und Teilfähigkeiten. Darüber hinaus zeigen sich aber auch Gemeinsamkeiten. So wird die Entwicklung der Rechtschreibkompetenz in den meisten Modellen als Abfolge von aufeinander aufbauenden Kompetenzen beschrieben. Auch inhaltlich gibt es Überschneidungen. Ausgehend von den Schreibprodukten, welche von den Kindern erstellt werden, lassen sich in allen Modellen folgende qualitative Unterschiede finden, welche im Folgenden als Kompetenzniveau bezeichnet werden sollen:

1. Es entstehen erste Schreibprodukte, wobei die Schreibungen noch nicht der Lautung eines Wortes entsprechen. Je nach Modell können dies erste gekritzelte Zeichen oder einzelne Buchstaben sein.

2. Es entstehen Schreibprodukte, die sich an der Lautung eines Wortes orientieren, sodass die Schreibung eines Wortes seiner Lautung entspricht.

3. Es entstehen korrekte Schreibungen, welche sich nicht ausschließlich über die Lautung des Wortes erklären lassen.

Im Vergleich zur Klarheit der inhaltlichen Abfolge der Entwicklung bleibt vage, wie Schülerinnen und Schüler einem Entwicklungsschritt zugeordnet werden, denn diese Information wird nur in 2 von 8 Modellen expliziert. Zusätzlich unterscheiden sich die Kriterien zwischen den Modellen. So basiert im Modell von Scheerer- 
Neumann (1989, 2015) die Zuordnung der Schülerinnen und Schüler zu einem Kompetenzlevel darauf, welche Schreibungen (und damit welche Strategie) mehrheitlich verwendet werden. Im Extremfall entscheidet somit ein einziges Wort über die Zuordnung zu einem Level. Günther (1986), Brügelmann und Brinkmann (1994), Thomé (2003) sowie May (1990, 2012) sprechen hingegen von der Dominanz einer Strategie bzw. Phase. Hierbei wird nur von May angegeben, was unter Dominanz zu verstehen ist. Fraglich ist bei allen Modellen, welchem Kompetenzniveau Schülerinnen und Schüler zugeordnet werden, die zwei oder mehr Strategien gleich häufig verwenden. Dies ist ein kritischer Punkt, da ein exaktes Zuordnungskriterium die Voraussetzung für die Zuordnung der Kinder zu einem Entwicklungsschritt und damit zur empirischen Überprüfung der theoretischen Modelle ist.

\section{Empirische Befunde zur Rechtschreibkompetenzentwicklung}

In bisherigen Längsschnittuntersuchungen zur Entwicklung der Rechtschreibkompetenz wurden am häufigsten standardisierte Rechtschreibtests angewendet, um anschließend zu zählen, wie viele Wörter die Schülerinnen und Schüler zu unterschiedlichen Messzeitpunkten korrekt geschrieben haben (vgl. z. B. Weinert und Helmke 1997; Mücke und Schründer-Lenzen 2008; Schneider 2008; Ditton und Krüsken 2009; Ennemoser et al. 2012; Fricke et al. 2015; Spika 2015; Stranghöner et al. 2017). Diese Studien konnten zeigen, dass Schülerinnen und Schüler von der Grundschule bis in die Sekundarstufe immer mehr Wörter richtig schreiben können.

Darüber hinaus existieren Studien, die eine genaue Aufschlüsselung von orthographischen Fehlern vornehmen. Hierbei werden Fehlerkategorien definiert, um anschließend zu prüfen, welche Fehler typisch für bestimmte Klassenstufen sind (z. B. Thomé und Gomolka 2007; Siekmann und Thomé 2012; Hoffmann-Erz 2015). Hoffmann-Erz (2015) beschreibt z. B. in ihrer Studie, dass lautliche Fehler, graphematische Abweichungen sowie Übergeneralsierungen mit zunehmender Klassenstufe seltener auftreten. Hierbei sei auch auf die vertiefenden Ergebnisse von IGLU-E verwiesen (Löffler und Meyer-Schepers 2005). Dort wurden auf Basis des zugrunde liegenden Modells von Löffler und Meyer-Schepers Fehler analysiert, die jeweils einer bestimmten Kompetenz zugeordnet werden konnten. Anschließend konnte gezeigt werden, welche Arten von Fehlern insbesondere Schülerinnen und Schüler mit Rechtschreibschwierigkeiten produzieren. In diesen Studien wurde nicht darauf eingegangen, inwiefern die Schülerinnen und Schüler individuell bestimmte Rechtschreibstrategien vorrangig verwendeten, sodass keine Rückschlüsse hinsichtlich des Kompetenzlevels möglich waren.

In den folgenden Studien wurde ein alternatives Vorgehen angewandt, um die verwendeten Rechtschreibstrategien zu berücksichtigen. Für die Auswertung der Vergleichsarbeiten (VERA 3, ISQ 2014) wurden die Rechtschreibleistungen von 5171 Schülerinnen und Schüler der dritten Jahrgangsstufe in Brandenburg einer von fünf Kompetenzstufen zugeordnet. Hierfür wurden 23 Aufgaben von den Schülerinnen und Schülern bearbeitet, in denen sowohl Wörter in Lückensätzen ergänzt werden mussten, als auch Aufgaben, in denen die richtige Schreibung ausgewählt oder begründet werden musste. Die Ergebnisse zeigten, dass $42 \%$ der Schülerinnen 
und Schüler überwiegend lautgetreu schrieben (Kompetenzstufe I). $24 \%$ der Schülerinnen und Schüler verwendeten erste Rechtschreibstrategien (Kompetenzstufe II), $20 \%$ waren in der Lage, Morpheme zur Gliederung von Wörtern zu beachten (Kompetenzstufe III). Die Kompetenzstufe IV, auf der nicht nur Wörter mit konkreter, sondern auch mit abstrakter Bedeutung richtig geschrieben werden, erreichten $7 \%$ der Schülerinnen und Schüler. Die höchste Stufe, auf der auch Wörter mit schwer zu durchschauenden Strukturen richtig geschrieben werden müssen, erreichten schließlich $7 \%$ der Schülerinnen und Schüler. Die VERA-Kompetenzstufen unterscheiden sich stark von den postulierten Kompetenzniveaus in den theoretischen Modellen zur Entwicklung der Rechtschreibkompetenz. Den Analysen liegen zudem ausschließlich Querschnittsdaten zugrunde. Diese können dazu dienen, die Rechtschreibkompetenzen der Schülerinnen und Schüler in der dritten Klassenstufe zu beschreiben, es können jedoch keine Aussagen über die Entwicklung der Schülerinnen und Schüler getroffen werden.

Scheerer-Neumann und Schnitzler (2009) untersuchten die Rechtschreibkompetenz von 178 Schülerinnen und Schülern vom Beginn der zweiten Klasse bis zum Beginn der dritten Klasse. Die Schreibungen wurden entweder als orthographisch korrekt, voll entfaltet alphabetisch (dies entspricht einer lautgetreuen Schreibung) oder beginnend alphabetisch kategorisiert. Anschließend bestimmten sie die prozentuale Verteilung der Schreibungen. Es zeigte sich, dass zu Beginn des zweiten Schuljahres $40 \%$ der Wörter orthographisch korrekt und weitere $31 \%$ lautgetreu geschrieben wurden (Scheerer-Neumann und Schnitzler 2009). Zusätzlich wurden auf Grundlage der Ergebnisse zum ersten Messzeitpunkt Leistungsgruppen gebildet. Es wurde deutlich, dass in jeder Gruppe Zuwächse innerhalb eines Schuljahres zu verzeichnen waren, wobei die größten Zuwächse in der Gruppe der schwächsten Schülerinnen und Schüler auftraten. So konnten Grundschulkinder, die zu Beginn der zweiten Klasse noch weniger als $30 \%$ der Wörter mindestens lautgetreu schrieben, zu Beginn der dritten Klasse mehr als $60 \%$ der Wörter mindestens lautgetreu schreiben (Scheerer-Neumann und Schnitzler 2009).

Auch wenn in den zuletzt genannten Studien die Rechtschreibkompetenz unter Berücksichtigung der verwendeten Strategie erhoben wurde, so wurde nicht geprüft, inwiefern Schülerinnen und Schüler individuell von niedrigeren Kompetenzniveaus zu höheren Kompetenzniveaus wechselten.

May (1993) untersuchte in seiner Studie die Schreibungen von 400 Schülerinnen und Schülern längsschnittlich von der ersten bis zur neunten Klasse. Es wurden fünf Leistungsgruppen ermittelt (I: gute Rechtschreiber ca. $25 \%$ der Stichprobe; II und III: durchschnittliche Rechtschreiber ca. 50\% der Stichprobe; IV: schwache Rechtschreiber $20 \%$ der Stichprobe und V: extrem schwache Rechtschreiber ca. $5 \%$ der Stichprobe). Anschließend wurden die Schreibungen eines Wortes innerhalb einer Gruppe hinsichtlich der einzelnen Grapheme verglichen. Das Ergebnis soll nach May (1993) die „typische Schreibung“ (May 1993) der Gruppe zeigen. Diese typische Schreibung der Gruppe wurde einer Rechtschreibstrategie zugeordnet. Dabei zeigte sich, dass alle Leistungsgruppen zunächst wesentliche Phoneme verschrifteten, dann immer mehr zu einer lautgetreuen Schreibung übergingen und schließlich orthographische Regeln berücksichtigten. Der Ablauf war in allen Leistungsgruppen gleich, der Zeitpunkt, zu dem die Gruppen einzelne Strategien beherrschten, vari- 
ierte jedoch. Hierbei wurde jedoch nur die am häufigsten vorkommende Schreibung der Gruppe erfasst. Individuelle Verläufe und ihre Variabilität wurden nicht näher untersucht.

Brinkmann (2015) untersuchte die Schreibung des Wortes Fahrrad von 924 Schülerinnen und Schülern der ersten bis vierten Klasse zu drei Terminen innerhalb von drei Wochen. Ihre Ergebnisse bestätigen insofern das Modell von May, indem die korrekte Schreibung des Wortes zunahm, während andere Schreibvarianten zurückgingen. Zusätzlich zeigten sich starke Schwankungen in den individuellen Schreibungen der Schülerinnen und Schüler. Die Autorin folgert daraus, dass die Schülerinnen und Schüler innerhalb des Erhebungszeitraums mehrere Strategien parallel anwenden. Dies widerspricht jedoch nicht dem Modell von May (1993). In diesem wird beschrieben, dass Schülerinnen und Schüler bestimmte Strategien dominant anwenden, wobei vorangegangene Strategien weiterhin verfügbar bleiben. Die Ergebnisse der Studie verdeutlichen zudem, wie wichtig die Erfassung der Rechtschreibkompetenz mit Hilfe von mehreren Wörtern ist, um die Schülerinnen und Schüler zuverlässig einem Entwicklungsschritt zuordnen zu können.

Bulut (2018) untersuchte die Rechtschreibleistung von 641 Schülerinnen und Schülern mit dem Ziel, den Verlauf der Rechtschreibkompetenz zu testen. Zu sechs Messzeitpunkten wurden jeweils drei Wörter verwendet, aus denen vier Lupenstellen gebildet wurden, welche das jeweilige Kompetenzniveau verdeutlichen soll: Für diese vier Lupenstellen wurde geprüft, wann ein Kind diese stabilisiert (Bulut 2018), d.h. ab wann diese von einem Kind richtig und anschließend nicht mehr falsch geschrieben wurde. Die Autorin berichtet, dass dies den gängigen Annahmen entspricht. Bulut untersuchte darüber hinaus, inwiefern die individuelle Entwicklung analog verläuft, wobei dies nicht der Reihenfolge entsprechen soll. Fraglich ist hierbei, inwiefern die Auswahl einzelner Lupenstellen ausreichend ist, um auf ein Kompetenzniveau zu schließen.

Zusammenfassend zeigt sich, dass in bisherigen Studien zumeist die Anzahl richtig geschriebener Wörter ermittelt wurde, spezifische Rechtschreibfehler untersucht wurden oder die geschriebenen Wörter einer Strategie zugeordnet wurden, nicht aber Schülerinnen und Schüler zu einem Niveau. Mit Hilfe der Vergleichsarbeiten (ISQ 2014) wurden Kompetenzstufen formuliert, die jedoch nicht ausschließlich auf Kriterien der Rechtschreibstrategien beruhen. Derzeit existieren nach unserem Kenntnisstand keine Studien, in denen die Entwicklung der Schülerinnen und Schüler entlang der dargestellten theoretischen Modelle empirisch überprüft wurde. Möglicherweise ist dies auch auf den fehlenden Konsens hinsichtlich der Zuordnungskriterien der Kinder zu einem Entwicklungsschritt zurückzuführen.

\section{Die vorliegende Untersuchung}

In der vorliegenden Untersuchung soll die postulierte hierarchische Struktur der Kompetenzniveaus des Rechtschreibens empirisch überprüft werden. Dabei werden die folgenden drei Kompetenzniveaus zugrunde gelegt: 1) nicht lautgetreu, 2) lautgetreu und 3) orthographisch korrekt. In der Literatur wird, wie zuvor beschrieben, nicht bei allen Modellen ein Zuordnungskriterium für die Kinder zu den einzelnen 
Niveaus angegeben, bzw. die angegebenen Kriterien variieren zwischen den Modellen. Statt ein Kriterium auszuwählen, soll geprüft werden, inwiefern sich die Gruppen auch in den Daten finden lassen. Um zudem die Wechsel zwischen den Gruppen berücksichtigen zu können, wurde die Rechtschreibkompetenz der Schülerinnen und Schüler zu drei Messzeitpunkten erhoben. Ziel ist es, die folgenden Fragen zu beantworten:

1. Inwiefern zeigen sich die Kompetenzniveaus empirisch?

Wenn die postulierten Kompetenzniveaus existieren, dann sollten sich die Schülerinnen und Schüler auf einem Niveau hinsichtlich ihrer Strategienutzung möglichst ähnlich sein. Dies sollte sich insofern in den Daten zeigen, dass sich Subgruppen identifizieren lassen, in denen die Strategienutzung zwischen den Schülerinnen und Schülern ähnlich ist. Es sollten sich demnach drei Hauptgruppen bilden lassen, die jeweils eine bestimmte Strategie bevorzugt verwenden.

H1 Es lassen sich drei Gruppen finden, die inhaltlich den Kompetenzniveaus entsprechen.

2. Wie verändert sich die Zuordnung der Schülerinnen und Schüler zu den Kompetenzniveaus über zwei Grundschuljahre hinweg?

$\mathrm{Zu}$ erwarten ist, dass die Schülerinnen und Schüler von niedrigeren Kompetenzniveaus zu höheren Kompetenzniveaus wechseln. Ein Wechsel von höheren Kompetenzniveaus zu niedrigeren würde demnach dem Modell widersprechen.

H2 Die Schülerinnen und Schüler wechseln von niedrigeren zu höheren Kompetenzniveaus.

\section{Methode}

\subsection{Stichprobe und Design}

Die vorliegende Studie ist Teil der längsschnittlichen Evaluationsstudie zum Projekt „Inklusive Grundschule“ (PING, Spörer et al. 2015). Das Ziel der Studie war herauszufinden, welche individuellen und kontextualen Bedingungen die akademischen und sozialen Entwicklungen von Schülerinnen und Schülern im Grundschulalter beeinflussen. Diese Studie bezieht sich auf längsschnittliche Daten aus der Testung der Schülerinnen und Schüler. Hierfür wurde die Rechtschreibkompetenz zu drei Messzeitpunkten (Anfang der 2. Klasse (t1), Ende der 2. Klasse (t2) und Ende der 3. Klasse (t3)) in 30 zufällig gezogenen Grundschulklassen untersucht. Insgesamt liegen Daten von $N=697$ Schülerinnen und Schülern vor $(\mathrm{t} 1=599, \mathrm{t} 2=606$, $\mathrm{t} 3=596)$, von denen 497 Schülerinnen und Schüler zu allen drei Messzeitpunkten an der Untersuchung teilnahmen. Darüber hinaus verließen Schülerinnen und Schüler frühzeitig die Stichprobe (von t1 zu t2: $n=44$ bzw. von t2 zu t3: $n=74$ ) bzw. wurden verspätet aufgenommen (von t1 zu t2: $n=51$ bzw. von t2 zu t3: $n=64$ ). Dabei bestand ein systematischer Dropout dahingehend, dass Schülerinnen und Schüler, die im Verlauf der gesamten Untersuchung in der Stichprobe verblieben, zu t1 signifikant mehr Wörter lautgetreu $(t=13,64, p<0,001)$ und signifikant mehr Wörter korrekt schrie- 
ben $(t=13,71, p<0,001)$ als Schülerinnen und Schüler, die vorzeitig die Stichprobe verließen. Auch hinsichtlich des Alters wird der Unterschied signifikant $(t=-2,71$, $p<0,05)$, sodass ältere Schülerinnen und Schüler häufiger die Stichprobe verlassen. Hinsichtlich Geschlecht $\left(\chi^{2}=1,82, p=0,18\right)$ unterschieden sich die Gruppen hingegen nicht signifikant. Für die Teilnahme an der Untersuchung wurde kein separates Einverständnis der Eltern benötig, da die Erhebungen im Rahmen einer landesweiten Evaluationsstudie zum inklusiven Lernen durchgeführt wurde.

$\mathrm{Zu}$ t1 waren die Schülerinnen und Schüler durchschnittlich 7,7 Jahre alt $(S D=0,46)$. Mädchen und Jungen waren etwa gleich häufig vertreten (50\% Mädchen). Fast alle Schülerinnen und Schüler wurden in Deutschland geboren. Lediglich $1 \%$ der Schülerinnen und Schüler gaben ein anderes Herkunftsland an, was für das Bundesland Brandenburg erwartungskonform war.

\subsection{Instrumente und Ablauf der Untersuchung}

$\mathrm{Zu}$ jedem Messzeitpunkt wurden die akademischen Kompetenzen im Klassenverband innerhalb von zwei Unterrichtsstunden erhoben, wobei für die Erfassung der Rechtschreibkompetenz ca. 30 min eingeplant waren. Zwischendurch wurden Lockerungsübungen gezeigt. Für Schülerinnen und Schüler, die Schwierigkeiten im Sehen oder den Förderschwerpunkt geistige Entwicklung hatten, standen Erhebungsmaterialien in Großdruck zur Verfügung. Dies erfolgte nach vorheriger Absprache mit den Lehrkräften. Die Teilnahme an der Untersuchung war freiwillig.

Zur Erfassung der Rechtschreibkompetenz wurde die Hamburger Schreibprobe (May 2012) verwendet. Diese liegt in verschiedenen Versionen für unterschiedliche Klassenstufen vor. Zu t1 wurde die HSP 1+ verwendet, zu t2 und t3 die HSP 2. Die Schülerinnen und Schüler sollten sowohl Einzelwörter als auch Sätze verschriften. Die Wörter sollten dabei von den Testleiterinnen und Testleitern gelesen und nicht diktiert werden, wobei die Wörter so oft vorgelesen wurden, wie dies von den Schülerinnen und Schülern benötigt wurde. Zu den Messzeitpunkten wurden jeweils die vollständige Version der HSP eingesetzt. Es ergaben sich für Gruppenanalysen ausreichend bis gute Reliabilitäten (Cronbachs $\alpha_{\mathrm{t} 1}=0,66, \alpha_{\mathrm{t} 2}=0,84, \alpha_{\mathrm{t} 3}=0,87$ ). Für die Beantwortung der vorliegenden Hypothesen wurden die Schreibungen sogenannter rechtschreibschwieriger Wörter betrachtet, d.h. es wurden nur jene Wörter ausgewertet, bei denen sich die lautgetreue Schreibung von der orthographisch korrekten Schreibweise unterschied. Daher wurde z.B. das Wort Mäuse in die Auswertung einbezogen, das Wort Baum hingegen nicht. Wörter, die bereits orthographisch korrekt waren, wenn sie lautgetreu geschrieben wurden, wurden für die Analysen nicht berücksichtigt. Für t1 wurden 11 Wörter ausgewählt, für t 2 und $\mathrm{t} 3$ konnten 17 Wörter mit orthografischen Schwierigkeiten identifiziert werden. Die Reliabilitäten der Skalen mit ausschließlich Wörtern mit orthographischen Schwierigkeiten fielen zu allen drei Messzeitpunkten zufriedenstellend bis gut aus (Cronbachs $\alpha_{\mathrm{t} 1}=0,69, \alpha_{\mathrm{t} 2}=0,84$, $\left.\alpha_{\mathrm{t} 3}=0,85\right)$. 


\subsection{Statistische Analysen}

Um die Fragestellung zu beantworten, wurden latente Transitionen berechnet. Ein wesentlicher Schritt war dabei die Zuordnung der Kinder zu den Strategien. Hierbei wurde wie folgt vorgegangen. Zunächst wurde für jedes Wort überprüft, ob die Schülerinnen und Schüler das Wort orthographisch korrekt verschriftet haben. Hierzu zählte auch die Groß- und Kleinschreibung der Wörter. Zusätzlich wurde für jede Schreibung eines Wortes von zwei unabhängigen Raterinnen und Ratern (in Anlehnung an Moll und Landerl 2010) eingeschätzt, inwiefern das Wort lautgetreu verschriftet wurde $\left(K_{\mathrm{t} 1}=0,83, K_{\mathrm{t} 2}=0,82, K_{\mathrm{t} 3}=0,85\right)$. Für jedes Wort entstanden so drei mögliche Kodierungen: orthographisch korrekt (2), lautgetreu (1) oder nicht lautgetreu (0). Anschließend wurden für jedes Grundschulkind Prozente gebildet, wie viel Prozent der Wörter die jeweilige Kodierung erhielten. Für jedes Grundschulkind wurden so zu jedem Messzeitpunkt drei Prozentangaben gebildet. Diese 9 Variablen bildeten die Analysevariablen der latenten Transitionsanalyse.

Zur Beantwortung der Fragestellung wurden latente Transitionsanalysen (LTA) unter Verwendung von Mplus (Muthén und Muthén 1998-2012) durchgeführt. Die LTA ist eine längsschnittliche Erweiterung der latenten Klassenanalyse. Diese ermöglicht es, die Gruppen so zu bilden, dass die Schülerinnen und Schüler innerhalb einer Gruppe möglichst homogen sind und gleichzeitig eine möglichst große Heterogenität zwischen den Gruppen existiert (Lanza und Cooper 2016). Hickendorff et al. (2018) sehen den Vorteil der latenten Tranisitionsanalyse vor allem auch darin, dass qualitative Unterschiede zwischen den Schülerinnen und Schülern identifiziert werden können. Dies ist für die Entwicklung entlang der Kompetenzniveaus entscheidend, da hierbei nicht nur die Anzahl der Fehler im Mittelpunkt steht, sondern auch, auf welchem Kompetenzniveau die Schülerinnen und Schüler schreiben können. Anschließend werden latente Übergangswahrscheinlichkeiten berechnet. Mplus verwendet hierfür standardmäßig den Maximum Likelihood Estimator mit robustem Standardfehler. Fehlende Werte wurden mittels Full Information Maximum Likelihood geschätzt. Hierfür lag die Covariance Coverage für die verwendeten Variablen zwischen 0,775 und 0,898 und liegt damit deutlich über dem mindestens geforderten Wert von 0,100. Die Anzahl zufälliger Starts wurde auf 400 und die Anzahl der Optimierungen auf 100 gesetzt, um lokale Maxima vermeiden zu können und die Lösung zuverlässiger abzusichern.

Ryoo et al. (2018) weisen darauf hin, dass in bisherigen Studien unterschiedliche Schritte für die Berechnungen von LTAs durchgeführt wurden. Ein großer Unterschied besteht dahingehend, inwiefern zunächst latente Klassen für jeden einzelnen Messzeitpunkt berechnet werden. Zeigen sich dieselben Gruppen zu jedem Messzeitpunkt, so soll dies sicherstellen, dass dieselben Gruppen über die Zeit hinweg existieren. Nun ist in der vorliegenden Studie jedoch aus theoretischer Sicht zu erwarten, dass die Gruppe der Schülerinnen und Schüler, die überwiegend nicht lautgetreu schreiben, zum Ende der dritten Klasse gering ausfällt. Hierdurch besteht die Befürchtung, dass diese Gruppe, obwohl sie inhaltlich relevant ist und in geringem Umfang auftreten könnte, von der latenten Klassenanalyse nicht erfasst wird. Aus diesem Grund werden die Profile anhand der latenten Transitionsanalyse definiert. $\mathrm{Zu}$ jedem Messzeitpunkt sollen bei der LTA gleich viele Gruppen existieren, 
welche inhaltlich gleich sind. Hierfür wurden die Mittelwerte der Gruppen zu t1 als Referenz für folgende Messzeitpunkte verwendet. Dies erleichtert die Interpretation der Gruppen. Trotzdem ist es möglich, dass sich die Anzahl der Schülerinnen und Schüler in den jeweiligen Gruppen verändert.

Für die Entscheidung, welche Klassenzuweisung verwendet werden soll, wurden folgende Kriterien herangezogen:

1. Der Entropy-Index soll möglichst groß sein. Werte der Entropy liegen zwischen 0 und 1 und zeigen an, wie präzise Personen den unterschiedlichen Klassen zugeordnet werden können.

2. Das Akaike information criterion (AIC), das Bayes information criterion (BIC) und das sample-size-adjusted BIC (SSA-BIC) dienen dazu, die Modelle hinsichtlich des Modellfits miteinander zu vergleichen (relativer Modellfit) und sollten möglichst klein sein (vgl. Henoch et al. 2015). Geiser (2010) weist darauf hin, dass ein Modell mit $N+1$ Klassen Daten tendenziell besser repräsentiert als ein Modell mit $N$ Klassen. Die Lösung sollte demnach mit möglichst wenigen Klassen auskommen (ebd.). Nach Masyn (2013) ist es möglich, dass die Kriterien zum relativen Modellfit sogar kein Minimum erreichen, sodass auch die Differenz zwischen den Modellen berücksichtigt werden muss. Hinzu kommt, dass möglicherweise die am besten statistisch passende Lösung inhaltlich wenig sinnvoll ist und deshalb für weitere Betrachtungen wenig brauchbar wäre (Nylund 2007).

3. Aus diesem Grund soll die Klassenlösung interpretierbar sein. Hierfür müssen sich die Mittelwerte zwischen den Profilen deutlich unterscheiden und die Streuungen innerhalb einer Klasse nicht zu groß sein. Weiterhin gehört dazu, dass die Profile zu jedem Messzeitpunkt ausreichend besetzt sind (vgl. Geiser 2010). Ziel ist es, dass sich die Profile inhaltlich unterscheiden.

Um Hypothese H2 zu überprüfen werden Übergangswahrscheinlichkeiten zwischen den gefundenen Profilen geprüft.

\section{Ergebnisse}

In Tab. 2 sind zunächst die deskriptiven Kennwerte zu den drei Messzeitpunkten dargestellt. Im Mittel wurden zu t1 ca. die Hälfte der Wörter lautgetreu geschrieben,

Tab. 2 Deskriptive Kennwerte und Stabilität der Variablen

\begin{tabular}{|c|c|c|c|c|c|c|}
\hline & \multicolumn{3}{|l|}{$M(S D)$} & \multicolumn{3}{|l|}{ Stabilität } \\
\hline & $\mathrm{t} 1$ & $\mathrm{t} 2$ & $\mathrm{t} 3$ & $r(\mathrm{t} 1-\mathrm{t} 2)$ & $r(\mathrm{t} 2-\mathrm{t} 3)$ & $r(\mathrm{t} 1-\mathrm{t} 3)$ \\
\hline Nicht lautgetreu & $\begin{array}{l}17,22 \\
(21,52)\end{array}$ & $\begin{array}{l}15,00 \\
(17,75)\end{array}$ & $\begin{array}{l}8,24 \\
(14,95)\end{array}$ & $0,68 *$ & $0,70 *$ & $0,62 *$ \\
\hline Lautgetreu & $\begin{array}{l}50,19 \\
(18,42)\end{array}$ & $\begin{array}{l}42,84 \\
(19,86)\end{array}$ & $\begin{array}{l}25,94 \\
(17,54)\end{array}$ & $0,41 *$ & $0,59 *$ & $0,14^{*}$ \\
\hline $\begin{array}{l}\text { Orthographisch } \\
\text { korrekt }\end{array}$ & $\begin{array}{l}32,60 \\
(17,68)\end{array}$ & $\begin{array}{l}42,16 \\
(24,53)\end{array}$ & $\begin{array}{l}65,82 \\
(24,06)\end{array}$ & $0,67 *$ & $0,75^{*}$ & $0,56^{*}$ \\
\hline
\end{tabular}

t1 Messzeitpunkt 1 (Anfang Klasse 2), $t 2$ Messzeitpunkt 2 (Ende Klasse 2), $t 3$ Messzeitpunkt 3 (Ende Klasse 3)

$* p<0,05$ 
Tab. 3 Indizes zur Modellgüte für Modelle mit unterschiedlichen Profilzahlen

\begin{tabular}{lllllll}
\hline $\begin{array}{l}\text { Anzahl } \\
\text { Profile }\end{array}$ & $\begin{array}{l}\text { Anzahl } \\
\text { Parameter }\end{array}$ & AIC & BIC & SSA-BIC & Entropy & $\begin{array}{l}\text { Log like- } \\
\text { lihood }\end{array}$ \\
\hline 2 & 20 & $46.163,83$ & $46.254,77$ & $46.191,27$ & 0,812 & $-23.061,92$ \\
3 & 32 & $44.162,65$ & $44.308,15$ & $44.206,55$ & 0,865 & $-22.049,33$ \\
4 & 48 & $43.106,14$ & $43.324,39$ & $43.171,98$ & 0,839 & $-21.505,07$ \\
5 & 68 & $42.323,35$ & $42.632,53$ & $42.416,61$ & 0,847 & $-21.093,67$ \\
6 & 92 & $41.845,86$ & $42.264,16$ & $41.972,04$ & 0,831 & $-20.830,93$ \\
7 & 120 & $41.521,39$ & $42.067,01$ & $41.685,98$ & 0,828 & $-20.640,70$ \\
8 & 152 & $41.234,93$ & $41.926,04$ & $41.443,41$ & 0,837 & $-20.465,47$ \\
9 & 188 & $40.938,01$ & $41.792,81$ & $41.195,87$ & 0,854 & $-20.281,01$ \\
10 & 228 & $40.719,44$ & $41.756,11$ & $41.032,16$ & 0,842 & $-20.131,72$ \\
\hline
\end{tabular}

Die jeweils günstigsten Werte sind kursiv markiert

$A I C$ Akaike information criterion, BIC Bayes information criterion, SSA-BIC sample-size-adjusted Bayesian information criterion

wobei bereits $37 \%$ der Wörter orthographisch korrekt geschrieben wurden. Der Anteil der orthographisch korrekt geschriebenen Wörter nimmt erwartungsgemäß zu t2 und t3 zu, wobei der Anteil nicht lautgetreuer und lautgetreuer Schreibungen abnimmt. Hinsichtlich der Stabilität wird deutlich, dass der Anteil nicht lautgetreuer und korrekter Schreibungen hohe Zusammenhänge zeigt, wohingegen vor allem der Anteil lautgetreuer Schreibungen zwischen den Messzeitpunkten nur gering korreliert.

Die erste Forschungsfrage bezieht sich darauf, inwiefern die Daten eine Clusterstruktur zeigen, die dem Kompetenzniveaumodell entspricht. Wie in Abschn. 5.3 beschrieben, werden hierfür alle Messzeitpunkte berücksichtigt. In Tab. 3 sind die Fit-Indices für Modelle mit 2 bis 10 Profilen dargestellt. Alle Modelle weisen eine Entropy größer als 0,8 auf, sodass die Schülerinnen und Schüler mit hoher Wahrscheinlichkeit genau einer Klasse zugeordnet werden konnten. Hierbei fällt die Entropy für das Modell mit 3 Klassen am höchsten aus. Bezüglich der Kenn-

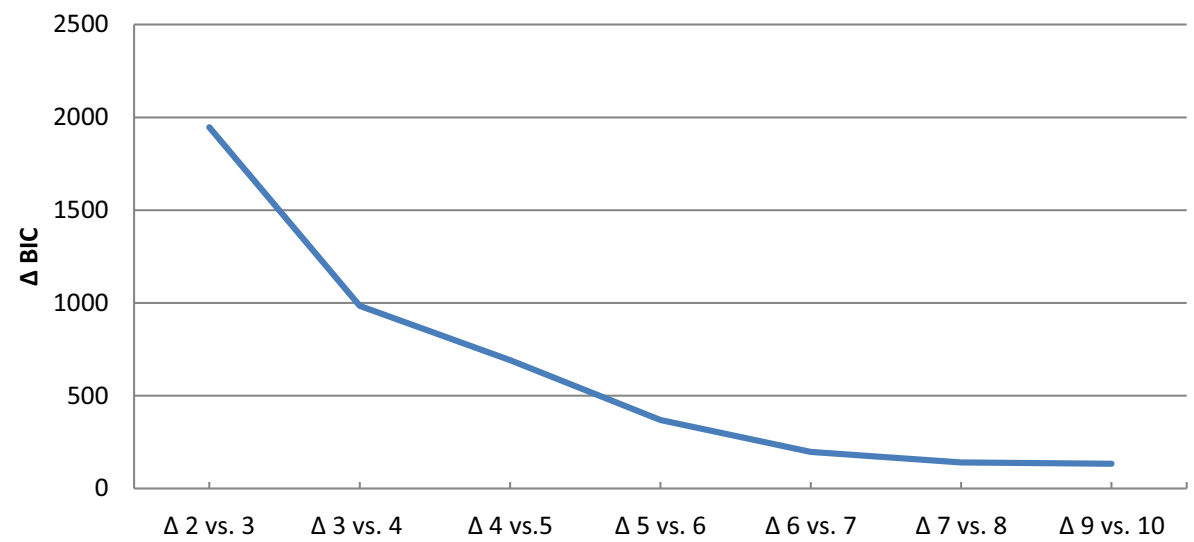

Abb. 1 Differenz des relativen Modellfits zwischen den Profillösungen 
Tab. 4 Kennwerte der einzelnen Gruppen der 3-Profillösung und Anteil der zugeordneten Schülerinnen und Schüler

\begin{tabular}{lllllllc}
\hline Profile & Bezeichnung & M zu t1 & \multicolumn{4}{c}{ Anteil der Stichprobe } \\
& & $\begin{array}{l}\text { Nicht } \\
\text { lautge- } \\
\text { treu }\end{array}$ & $\begin{array}{l}\text { Laut- } \\
\text { getreu }\end{array}$ & $\begin{array}{l}\text { Orthogra- } \\
\text { phisch } \\
\text { korrekt }\end{array}$ & & t2 & t3 \\
\hline 1 & Buchstabenentdeckende & 66,39 & 22,71 & 10,91 & 12,20 & 9,47 & 6,60 \\
2 & Phonemprofis & 11,58 & 56,63 & 31,80 & 80,34 & 61,26 & 18,22 \\
3 & Rechtschreibspezialisten & 4,90 & 26,87 & 68,24 & 7,46 & 29,27 & 75,18 \\
\hline
\end{tabular}

werte zum relativen Modellfit (AIC, BIC und SSA-BIC) wird deutlich, dass diese mit steigender Klassenzahl immer kleiner werden.

Masyn (2013) empfiehlt für die Betrachtung der Differenz des relativen Modellfits Elbow-Plots (ähnlich zur Faktorenanalyse). Hierbei wird deutlich, dass der größte Zugewinn hinsichtlich des Modellfits bei der 3 Klassenlösung zu sehen ist (siehe Abb. 1).

Aus statistischer Sicht gibt es somit keine eindeutige Lösung, sodass die Entscheidung zwischen der 3-Profil-Lösung und der 6-Profil-Lösung auf Grundlage inhaltlicher Überlegungen getroffen werden muss.

In Tab. 4 sind die Mittelwerte der einzelnen Profile der 3-Profil-Lösung dargestellt. Zusätzlich ist dargestellt, welcher Anteil der Schülerinnen und Schüler zu den jeweiligen Messzeitpunkten den Profilen angehören. Die Mittelwerte der jeweiligen Profile wurden im Modell über die drei Messzeitpunkte konstant gehalten. Inhaltlich zeigen sich in der 3-Profillösung die postulierten Kompetenzniveaus. So schreiben Schülerinnen und Schüler im Profil 1 den überwiegenden Anteil der Wörter nicht lautgetreu (im Folgenden Buchstabenentdeckende). Profil 2 zeichnet sich hingegen dadurch aus, dass die Mehrheit der Wörter lautgetreu geschrieben wurden (im Folgenden Phonemprofis). Schülerinnen und Schüler in Profil 3 konnten bereits durchschnittlich $68 \%$ der Wörter korrekt verschriften (im Folgenden Rechtschreibspezialisten).

Betrachtet man diese Aspekte auch für die 6-Profil-Lösung, so wird zum einen deutlich, dass die Profile teilweise sehr gering besetzt sind (unter 5\% der Stichprobe). Bezüglich der Mittelwerte der einzelnen Gruppen ergeben sich zweimal zwischen zwei Profilen große Ähnlichkeiten. Sowohl Profil 4 als auch Profil 5 zeigen jeweils einen hohen Anteil korrekt geschriebener Wörter, einen geringen Anteil lautgetreu geschriebener Wörter und kaum Wörter, die nicht-lautgetreu geschrieben wurden. Dies kann als quantitative Unterschiede hinsichtlich des Profils der Rechtschreibspezialisten interpretiert werden. Analog finden sich die Profile 2 und 3, welche als Varianten für das Profil der Phonemprofis interpretiert werden können. Das Profil 1 der 6-Profil-Lösung entspricht inhaltlich dem der Buchstabenentdeckenden, sodass lediglich das Profil 6 inhaltlich von der 3-Profil-Lösung abweicht. Bei diesem Profil gibt es sowohl einen hohen Anteil nicht lautgetreu geschriebener Wörter und einen hohen Anteil lautgetreu geschriebener Wörter. Zu t1 wurden diesem Profil rund $13 \%$ der Schülerinnen und Schüler zugeteilt, wobei der Anteil zu den folgenden Messzeitpunkten sinkt, sodass zu t3 nur noch $4 \%$ der Schülerinnen und Schüler diesem Profil zugeordnet wurden. Im Sinne der besseren Interpretierbarkeit 
Tab. 5 Theoriekonforme Pfade, Anteil der Schülerinnen und Schüler, die diese durchlaufen haben sowie die standardisierten Ergebnisse der richtig geschriebenen Wörter der HSP zu den drei Messzeitpunkten

\begin{tabular}{|c|c|c|c|c|c|c|c|c|c|c|c|}
\hline Pfad & $\begin{array}{l}\text { Profil } \\
\mathrm{zu} \\
\mathrm{t} 1\end{array}$ & $\begin{array}{l}\text { Profil } \\
\text { zu } \\
\text { t2 }\end{array}$ & $\begin{array}{l}\text { Profil } \\
\text { zu } \\
\text { t3 }\end{array}$ & $N$ & Anteil & $\begin{array}{l}M \\
\text { T-Wert } \\
\text { zu t1 }\end{array}$ & $\begin{array}{l}S D \\
\text { T-Wert } \\
\text { zu t1 }\end{array}$ & $\begin{array}{l}M \\
\text { T-Wert } \\
\text { zu t2 }\end{array}$ & $\begin{array}{l}S D \\
\text { T-Wert } \\
\text { zu t3 }\end{array}$ & $\begin{array}{l}M \\
\text { T-Wert } \\
\text { zu t3 }\end{array}$ & $\begin{array}{l}S D \\
\text { T-Wert } \\
\text { zu t3 }\end{array}$ \\
\hline \multicolumn{12}{|c|}{ Theoriekonforme Pfade } \\
\hline 1 & $\mathrm{P}$ & $\mathrm{P}$ & $\mathrm{R}$ & 297 & 42,61 & 45,07 & 6,22 & 48,26 & 5,12 & 60,09 & 5,23 \\
\hline 2 & $\mathrm{P}$ & $\mathrm{R}$ & $\mathrm{R}$ & 151 & 21,66 & 48,64 & 5,54 & 57,65 & 3,50 & 64,64 & 5,13 \\
\hline 3 & $\mathrm{P}$ & $\mathrm{P}$ & $\mathrm{P}$ & 103 & 14,78 & 41,25 & 6,68 & 42,90 & 5,69 & 47,85 & 5,68 \\
\hline 4 & $\mathrm{R}$ & $\mathrm{R}$ & $\mathrm{R}$ & 52 & 7,46 & 59,62 & 4,53 & 61,69 & 5,91 & 69,74 & 5,46 \\
\hline 5 & B & B & B & 41 & 5,88 & 25,31 & 7,05 & 28,60 & 4,67 & 29,14 & 6,03 \\
\hline 6 & B & $\mathrm{P}$ & $\mathrm{R}$ & 15 & 2,15 & 34,33 & 7,31 & 41,50 & 6,06 & 54,33 & 3,91 \\
\hline 7 & B & B & $\mathrm{P}$ & 13 & 1,87 & 30,82 & 5,06 & 35,92 & 4,56 & 42,69 & 5,77 \\
\hline 8 & B & $\mathrm{P}$ & $\mathrm{P}$ & 9 & 1,29 & 32,89 & 6,53 & 39,78 & 3,56 & 44,67 & 5,00 \\
\hline 9 & B & B & $\mathrm{R}$ & 5 & 0,72 & 39,00 & 2,31 & 41,60 & 4,28 & 54,00 & 4,90 \\
\hline 10 & B & $\mathrm{R}$ & $\mathrm{R}$ & 1 & 0,14 & 33,00 & - & 53,00 & - & 64,00 & - \\
\hline
\end{tabular}

$B$ Buchstabenentdeckende, $P$ Phonemprofis, $R$ Rechtschreibspezialisten

und der Ökonomie wird für die nachfolgenden Analysen daher die 3-Profillösung betrachtet.

Im nächsten Schritt sollte geprüft werden, inwiefern die Schülerinnen und Schüler von niedrigen zu höheren Kompetenzniveaus wechseln. Hierfür wurde in Mplus die Profilzugehörigkeit zu den verschiedenen Messzeitpunkten für jedes Grundschulkind ermittelt, um anschließend die entstehenden Pfade zu bestimmen. Mit den drei entstandenen Profilen und drei Messzeitpunkten sind kombinatorisch 27 Pfade denkbar, welche von den Kindern durchlaufen werden können. In Tab. 5 sind die 10 theoriekonformen Pfade dargestellt, wobei sie danach geordnet wurden, von wie vielen Schülerinnen und Schülern sie durchlaufen wurden. Insgesamt durchliefen 687 Schülerinnen und Schüler theoriekonforme Pfade. Dies entspricht 98,57\% der Stichprobe. Bei 15 Schülerinnen und Schülern konnte dabei die gesamte Entwicklung von nicht lautgetreuem Schreiben über lautgetreues Schreiben zu korrektem Schreiben beobachtet werden. Ein Großteil der Schülerinnen und Schüler konnte bereits zu t1 den Phonemprofis zugeordnet werden. So wurden 297 Schülerinnen und Schüler zu t 1 und $\mathrm{t} 2$ den Phonemprofis zugeordnet und wechselten zu t 3 zu den Rechtschreibspezis. Weitere 151 Schülerinnen und Schüler, die zu t1 überwiegend lautgetreu schrieben, wechselten $\mathrm{zu} \mathrm{t} 2 \mathrm{zu}$ den Rechtschreibspezis und verblieben dort auch zu t3. Weitere 52 Schülerinnen und Schüler konnten bereits zu t1 den Rechtschreibspezis zugeordnet werden und verblieben zu t2 und $\mathrm{t} 3$ in dieser Gruppe. Insgesamt 85 Schülerinnen und Schüler wurden zu t1 der Gruppe der Buchstabenentdeckenden zugeordnet. Von diesen verblieben 41 Schülerinnen und Schülern $\mathrm{zu}$ t2 und $\mathrm{t} 3$ in dieser Gruppe. Weitere 13 Schülerinnen und Schüler wurden sowohl zu t1, als auch zu t2 den Buchstabenentdeckenden zugeordnet und wechselten dann zu t3 zu den Phonemprofis. Weitere 9 Schülerinnen und Schüler, die zu t1 der Gruppe der Buchstabenentdeckenden zugeordnet wurden, wechselten $\mathrm{zu} \mathrm{t} 2 \mathrm{zu}$ den Phonemprofis und verblieben zu t3 dort. 6 Schülerinnen und Schüler übersprungen das lautgetreue Schreiben, d.h. sie wurden zu t1 den Buchstabenentdeckenden 
Tab. 6 Übergangswahrscheinlichkeiten zwischen den Messzeitpunkten in Prozent

\begin{tabular}{llllllll}
\hline Profil & \multicolumn{3}{c}{ Von t1 zu t2 } & \multicolumn{4}{c}{ Von t2 zu t3 } \\
& & 1 & 2 & 3 & 1 & 2 & 3 \\
\hline 1 & Buchstabenentdeckende & 0,60 & 0,38 & 0,02 & 0,47 & 0,34 & 0,19 \\
2 & Phonemprofis & 0,02 & 0,69 & 0,29 & 0,01 & 0,32 & 0,67 \\
3 & Rechtschreibspezialisten & 0,00 & 0,00 & 1,00 & 0,00 & 0,00 & 1,00 \\
\hline
\end{tabular}

zugeordnet und wechselten zu t2 bzw. zu t3 zu den Rechtschreibspezis. Zur Illustration der Leistung der gefundenen Teilstichproben wurden in der Tabelle zusätzlich die Mittelwerte und Standardabweichungen der T-Werte für die Anzahl der richtig geschriebenen Wörter in der jeweiligen HSP dargestellt. Hierbei zeigt sich, dass die Zuordnung zu einem Profil, basierend auf den rechtschreibschwierigen Wörtern, mit den Rechtschreibleistungen, basierend auf allen geschriebenen Wörtern der HSP, korrespondiert. Befinden sich die Schülerinnen und Schüler auf einem höheren Kompetenzniveau, so erreichen sie auch insgesamt höhere T-Werte. Schülerinnen und Schüler, welche z.B. den Pfad 4 durchlaufen haben, wurden zu jedem Messzeitpunkt den Rechtschreibspezialisten zugeordnet und zeigen im Vergleich zu den anderen Schülerinnen und Schülern stets die höchsten T-Werte. Hingegen erzielen jene Schülerinnen und Schüler, welche den Pfad 5 durchlaufen haben (und somit zu allen Messzeitpunkten den Buchstabenentdeckenden zugeordnet wurden), zu allen drei Messzeitpunkten im Vergleich die niedrigsten T-Werte.

Kombinatorisch waren 17 nicht theorie-konforme Pfade möglich, welche mindestens einen Wechsel von höheren zu niedrigeren Profilen zwischen den Messzeitpunkten enthält. Lediglich 5 dieser Pfade wurden in der Stichprobe gefunden, wobei diese von insgesamt 10 Schülerinnen und Schülern durchlaufen wurden.

Um die Ergebnisse weiter abzusichern, wurden zusätzlich die Übergangswahrscheinlichkeiten zwischen den Gruppen berechnet. Diese sind in Tab. 6 dargestellt. Hinsichtlich der Übergangswahrscheinlichkeiten wird deutlich, dass alle Schülerinnen und Schüler, die zu t1 bereits zu den Rechtschreibspezialisten gehören, auch zu späteren Messzeitpunkten diesem Profil zugeordnet wurden. Deutlich wird auch, dass die Wahrscheinlichkeit von höheren zu niedrigeren Profilen $\mathrm{zu}$ wechseln $\mathrm{zu}$ beiden Messzeitpunkten unter 2\% liegt. Zwischen den ersten beiden Messzeitpunkten ist die Wahrscheinlichkeit für Kinder in den Profilen Buchstabenentdeckende bzw. Phonemprofis zu verbleiben, am höchsten. Wechsel zum nächst höheren Profil erreichen eine Wahrscheinlichkeit von 0,293 bis 0,375. Ein Wechsel von den Buchstabenentdeckenden zu Rechtschreibspezialisten, was ein Überspringen des lautgetreuen Niveaus bedeuten würde, ist von $\mathrm{t} 1 \mathrm{zu}$ t 2 sehr unwahrscheinlich. Dies ändert sich von $\mathrm{t} 2 \mathrm{zu}$ t3. Hier wechseln die Kinder von $\mathrm{t} 1 \mathrm{zu}$ t2 von den Buchstabenentdeckenden mit einer Wahrscheinlichkeit von 0,341 zu den Phonemprofis, aber auch mit 0,189 zu den Rechtschreibspezialisten. Kinder, die zu t2 den Phonemprofis zugeordnet wurden, wechseln am wahrscheinlichsten zu den Rechtschreibspezialisten. 


\section{Diskussion}

\subsection{Zusammenfassung der Befunde}

Wie entwickelt sich die Rechtschreibkompetenz von Grundschulkindern? Die Entwicklung der Schülerinnen und Schüler wird in theoretischen Modellen unterschiedlicher Autoren sehr ähnlich dargestellt. Betrachtet man jedoch die empirischen Befunde, so können diese theoretischen Modelle noch nicht als gesichert gelten. Die vorliegende Studie versuchte diese Forschungslücke zu bearbeiten.

Es sollte betrachtet werden, inwiefern sich die theoretisch postulieren Kompetenzniveaus auch in empirischen Daten wiederfinden lassen und inwiefern sich die $\mathrm{Zu}$ ordnung der Schülerinnen und Schüler zu den Kompetenzniveaus über zwei Grundschuljahre hinweg verändert. Es ergaben sich zwei Hauptbefunde. Erstens konnten die Schülerinnen und Schüler basierend auf ihren Rechtschreibleistungen drei Profilen zugeordnet werden, die inhaltlich den Kompetenzniveaus entsprachen, sodass die erste Hypothese bestätigt werden konnte.

Zweitens, zeigte sich, dass die Wechsel zwischen den Profilen, wie theoretisch vermutet, nahezu ausschließlich von niedrigeren zu höheren Kompetenzniveaus erfolgten. Die Übergangswahrscheinlichkeiten von höheren zu niedrigeren Profilen lag stets unter $2 \%$. Die Interpretation der T-Werte, basierend auf den jeweiligen Gesamt-HSP, unterstützt zusätzlich die gefundenen Profile, indem höhere Niveaus mit höheren Rechtschreibleistungen einhergehen.

Bereits Bulut (2018) konnte zeigen, dass sich aufeinander aufbauende Kompetenzen finden lassen. Dieses Ergebnis wird durch die vorliegende Studie gestärkt, insbesondere da hierbei eine größere Datenbasis (im Sinne von mehr geschriebenen Wörtern) zugrunde. Abweichend von Bulut wird jedoch auch für die individuelle Entwicklung der Schülerinnen und Schüler diese Entwicklung entlang der Kompetenzniveaus gefunden. Ein weiterer Unterschied besteht in der Art und Anzahl der untersuchten Kompetenzen. So spricht Bulut (2018) von drei Prinzipien (phonologisches, morphematisches und silbisches Prinzip), wobei in der Untersuchung selbst hierfür vier Lupenstellen verwendet wurden. Die vorliegende Arbeit unterscheidet jedoch die Kompetenzniveaus hinsichtlich nicht lautgetreu, lautgetreu und korrekt. Die Ergebnisse unterscheiden sich somit dahingehend, wie die Schreibung der Wörter gewertet werden.

Auch May (1993) behauptete mit seiner Studie die Stufenfolge zeigen zu können, betrachtete dabei jedoch die Entwicklung von Leistungsgruppen. Seine Ergebnisse können somit durch aktuelle Daten mit Berücksichtigung der individuellen Schreibungen der Schülerinnen und Schüler bestätigt werden. Dabei ist jedoch zu beachten, dass in der vorliegenden Studie nicht zwischen dem orthographischen und dem morphematischen Kompetenzlevel unterschieden werden kann.

Weiterhin finden sich Unterschiede in Hinblick auf den Zeitpunkt, wann die Schülerinnen und Schüler eine Kompetenz beherrschen. So findet sich bei May (1993) Mitte der 2. Klasse noch keine Gruppe, die die orthographische Strategie beherrscht. Zusätzlich ist in der leistungsschwächsten Gruppe die häufigste Schreibung bereits am Ende der 2. Klasse der alphabetischen Strategie zugeordnet. Durch die individuelle Betrachtung in der vorliegenden Studie konnte gezeigt werden, dass die 
Heterogenität der Schülerinnen und Schüler in Bezug auf die Kompetenzniveaus noch größer ist, als durch die Studie von May (1993) ersichtlich wird. So traten zu allen drei Messzeitpunkten alle drei Kompetenzniveaus auf. Dies ist möglicherweise darauf zurück zu führen, dass in der vorliegenden Stichprobe inklusive Schulklassen untersucht wurden. In diesen kann die Heterogenität durch die Aufnahme von Kindern mit unterschiedlichen Förderschwerpunkten erhöht sein. May (1993) kam zu dem Ergebnis, dass die Entwicklung der Rechtschreibkompetenz von Schülerinnen und Schülern qualitativ ähnlich, jedoch unterschiedlich schnell verläuft. Es könnte demnach so sein, dass die Schülerinnen und Schüler, die in der vorliegenden Studie zum dritten Messzeitpunkt noch auf dem niedrigsten Kompetenzniveau verharrten, später zu höheren Kompetenzniveaus wechseln. Um dies zu prüfen, wären Untersuchungen eben dieser Schülerinnen und Schüler über die dritte Jahrgangsstufe hinaus nötig gewesen.

\subsection{Limitationen}

Die erste Limitation liegt darin, dass zu den unterschiedlichen Messzeitpunkten unterschiedliche Wörter geschrieben werden mussten. So mussten Schülerinnen und Schüler zu t2 und $\mathrm{t} 3$ nicht nur mehr Wörter, sondern auch schwierigere Wörter schreiben. Dieser Wechsel zu schwierigeren Wörtern könnte somit erklären, wieso einige Schülerinnen und Schüler auf ihrem Kompetenzniveau verharrten. Derzeit existiert jedoch kein Rechtschreibtest, der jahrgangsübergreifend mit ein und denselben Wörtern verwendet werden kann. Für zukünftige Forschung, aber auch für die Diagnose der Rechtschreibkompetenz in der Praxis wäre die Entwicklung entsprechender Testverfahren hilfreich.

Eine weitere Limitation ist der systematische Drop-Out, sodass insbesondere Schülerinnen und Schüler die Stichprobe verließen, die zu Beginn der Untersuchung den überwiegenden Teil der Wörter nicht lautgetreu verschrifteten. Zwar wurden statistische Prozeduren verwendet, die mit den fehlenden Werten angemessen umgehen, die Entwicklung der Schülerinnen und Schüler, die die Stichprobe verließen, kann daher nur geschätzt werden. Eine individuelle Nachverfolgung dieser Schülerinnen und Schüler könnte somit darüber Aufschluss geben, inwiefern sich ihre Kompetenz tatsächlich entwickelt.

In der vorliegenden Studie können keine Rückschlüsse gezogen werden, inwiefern die unterschiedlichen Wechsel zwischen den Profilen auf bestimmte Lehrmethoden zurück zu führen sind. In zukünftigen Studien sollte überprüft werden, inwiefern es systematische Unterschiede zwischen den Klassen gibt und inwiefern diese auf Merkmale des Unterrichts zurück zu führen sind. Hierbei wäre es wünschenswert, wenn Schulen aus unterschiedlichen Bundesländern berücksichtigt werden können, um auszuschließen, dass die Ergebnisse durch unterschiedliche Lehrpläne oder regionale Dialekte beeinflusst werden.

Eine weitere Limitation bezieht sich auf die Zeitpunkte und Abstände zwischen den Untersuchungen. Zu Beginn der Untersuchung konnten viele Schülerinnen und Schüler bereits lautgetreu und einige bereits korrekt schreiben. Zukünftige Studien sollten daher noch in früheren Jahrgängen mit der Untersuchung zu beginnen, sodass besser untersucht werden kann, inwiefern die gesamte Entwicklung vollzogen wird. 
Zwischen zwei Messzeitpunkten lag zudem jeweils ca. ein Schuljahr. Dies ist im Schulkontext eine lange Zeit, in der die Kinder viel dazu lernen können. Zukünftige Studie sollten daher kleine Abstände der Untersuchungen wählen. Dadurch wäre es möglich zu prüfen, inwiefern die unterschiedliche Unterteilung der Kompetenzniveaus in den theoretischen Modellen zutreffend ist. Weiterhin könnte durch geringeren Abstände die Stabilität der Kompetenzniveaus besser geprüft werden. In der vorliegenden Studie wurden Pfade, welche von höheren zu niedrigeren Profilen verliefen, nur von zehn Schülerinnen und Schülern durchlaufen. Möglicherweise wäre dies bei geringeren Abständen zwischen den Messzeitpunkten häufiger aufgetreten.

\subsection{Fazit und Implikationen für die Praxis}

Die Ergebnisse verdeutlichen, dass Schülerinnen und Schüler von niedrigeren zu höheren Kompetenzniveaus wechseln. Die unterschiedlichen Kompetenzniveaus sind durch unterschiedliche Fehlerschwerpunkte gekennzeichnet. Diese Fehler sollen kein Ausdruck dafür sein, dass Schülerinnen und Schüler nicht richtig schreiben können. Vielmehr sind sie ein Ausdruck von ,entwicklungsgemäßen Fehlern“ (Schründer-Lenzen 2013, S. 70). Schreibungen, die sich zwar an der Aussprache orientieren, aber noch nicht der Normschreibung entsprechen sollten deswegen nicht unterbunden werden, sondern stattdessen als Durchgangsstadium im Entwicklungsprozess toleriert werden (vgl. Schründer-Lenzen 2013). Das Wissen über diese Fehlerschwerpunkte kann Lehrpersonen dabei unterstützen, den Lernstand der Schülerinnen und Schüler besser einzuschätzen, um dann solche Inhalte den Schülerinnen und Schülern näher zu bringen, welche sie als nächstes lernen können. Außerdem wird deutlich, dass sich die Schülerinnen und Schüler unterschiedlich schnell entwickeln, sodass einige bereits zu Beginn der zweiten Klasse die Mehrheit der Wörter orthographisch korrekt schreiben. Die Lehrerinnen und Lehrer haben somit die Aufgabe, den unterschiedlichen Entwicklungsniveaus der Schülerinnen und Schüler gerecht zu werden und durch Maßnahmen der inneren Differenzierung eine individuelle Förderung zu gewährleisten.

Die Ergebnisse zeigten auch markante Unterschiede hinsichtlich der Entwicklungsgeschwindigkeiten. Hier können zukünftige Studien ansetzten, um Bedingungen für besonders günstige Entwicklungen heraus zu finden.

Die latenten Transitionsanalysen konnten die theoretisch vermuteten Kompetenzniveaus stützen. Die gefundenen Gruppen unterschieden sich hinsichtlich ihrer T-Werte, sodass Schülerinnen und Schüler im Profil Buchstabenentdeckende die vergleichsweise schwächeren Rechtschreibleistungen zeigten, die Schülerinnen und Schüler im Profil Rechtschreibspezialisten hingegen die vergleichsweise stärksten Rechtschreibleistungen. Durch die latenten Transitionsanalysen konnte darauf verzichtet werden, ein absolutes Kriterium festzulegen, welches in den theoretischen Modellen kaum gegeben ist. Es konnte gezeigt werden, dass Schülerinnen und Schüler von niedrigeren zu höheren Kompetenzniveaus wechseln. Es bleibt jedoch offen, welche Merkmale diese Entwicklung beeinflussen, sodass dies in zukünftigen Untersuchungen näher betrachtet werden sollte. 
Funding Open Access funding provided by Projekt DEAL.

Open Access Dieser Artikel wird unter der Creative Commons Namensnennung 4.0 International Lizenz veröffentlicht, welche die Nutzung, Vervielfältigung, Bearbeitung, Verbreitung und Wiedergabe in jeglichem Medium und Format erlaubt, sofern Sie den/die ursprünglichen Autor(en) und die Quelle ordnungsgemäß nennen, einen Link zur Creative Commons Lizenz beifügen und angeben, ob Änderungen vorgenommen wurden.

Die in diesem Artikel enthaltenen Bilder und sonstiges Drittmaterial unterliegen ebenfalls der genannten Creative Commons Lizenz, sofern sich aus der Abbildungslegende nichts anderes ergibt. Sofern das betreffende Material nicht unter der genannten Creative Commons Lizenz steht und die betreffende Handlung nicht nach gesetzlichen Vorschriften erlaubt ist, ist für die oben aufgeführten Weiterverwendungen des Materials die Einwilligung des jeweiligen Rechteinhabers einzuholen.

Weitere Details zur Lizenz entnehmen Sie bitte der Lizenzinformation auf http://creativecommons.org/ licenses/by/4.0/deed.de.

\section{Literatur}

Blatt, I., \& Jarsinski, S. (2009). Auswertung nach der Sprachsystematischen Rechtschreibdiagnose. In R. Valtin \& B. Hofmann (Hrsg.), Kompetenzmodelle der Orthographie. Empirische Befunde und förderdiagnostische Möglichkeiten (S. 91-113). Berlin: Deutsche Gesellschaft für Lesen und Schreiben.

Brinkmann, E. (2015). Wie eignen sich Kinder die Rechtschreibung an? In E. Brinkmann (Hrsg.), Rechtschreiben in der Diskussion - Schriftspracherwerb und Rechtschreibunterricht. Beiträge zur Reform der Grundschule (S. 164-174). Frankfurt a. M.: Grundschulverband.

Brügelmann, H., \& Brinkmann, E. (1994). Stufen des Schriftspracherwerbs und Ansätze zu seiner Förderung. In H. Brügelmann \& S. Richter (Hrsg.), Wie wir recht schreiben lernen (S. 44-52). Lengwil: Libelle.

Bulut, N. (2018). Individuelle Rechtschreibentwicklung. Eine Längsschnittuntersuchung zur Bedeutung von Einflussfaktoren auf die Wortschreibung. Baltmannsweiler: Schneider Verlag Hohengehren.

Ditton, H., \& Krüsken, J. (2009). Denn wer hat, dem wird gegeben werden? Eine Längsschnittstudie zur Entwicklung schulischer Leistungen und den Effekten der sozialen Herkunft in der Grundschulzeit. Journal for Educational Research Online, 1(1), 33-61.

Eichler, W. (1976). Zur linguistischen Fehleranalyse von Spontanschreibungen bei Vor- und Grundschulkindern. In A. Hofer (Hrsg.), Lesenlernen: Theorie und Unterricht (S. 246-264). Düsseldorf: Pädagogischer Verlag Schwann.

Eichler, W. (1992). Schreibenlernen: Schreiben-rechtschreiben-Texte-Verfassen. Bochum: Ferdinand Kamp.

Ennemoser, M., Marx, P., Weber, J., \& Schneider, W. (2012). Spezifische Vorläuferfertigkeiten der Lesegeschwindigkeit, des Leseverständnisses und des Rechtschreibens. Evidenz aus zwei Längsschnittstudien vom Kindergarten bis zur 4. Klasse. Zeitschrift für Entwicklungspsychologie und Pädagogische Psychologie, 44(2), 53-67.

Fricke, S., Szczerbinski, M., Fox-Boyer, A., \& Stackhouse, J. (2015). Preschool predicotrs of early literacy acquisition in German-speaking children. Reading Research Quarterly, 51(1), 29-53.

Frith, U. (1985). Beneath the surface of developmental dyslexia. In K. Patterson, J. C. Marshall \& M. Coltheart (Hrsg.), Surface dyslexia: Neuropsychological and cognitive studies of phonological reading (S. 301-330). London: Erlbaum.

Geiser, C. (2010). Datenanalyse mit Mplus. Eine anwendungsorientiere Einführung. Wiesbaden: VS.

Günther, K.B. (1986). Ein Stufenmodell der Entwicklung kindlicher Lese- und Schreibstrategien. In H. Brügelmann (Hrsg.), ABC und Schriftsprache: Rätsel für Kinder, Lehrer und Forscher (S. 32-54). Konstanz: Faude.

Henoch, J., Klusman, U., Lüdtke, O., \& Trautwein, U. (2015). Die Entwicklung beruflicher Selbstregulation: Ein Vergleich zwischen angehenden Lehrkräften und anderen Studierenden. Zeitschrift für Pädagogische Psychologie, 29(3-4), 151-162. 
Hickendorff, M., Edelsbrunner, P. A., McMulle, J., Schneider, M., \& Trezise, K. (2018). Informative tools for characterizing individual differences in learning: Latent class, latent profile, and latent transition analysis. Learning and Individual Differences, 66, 4-15.

Hoffmann-Erz, R. (2015). Lernprozesse im Orthographiewerwerb. Eine empirische Studie zur Entwicklung der Generalisierungskompetenz. Berlin: Wissenschaftlicher Verlag Berlin.

ISQ (2014) = Institut für Schulqualität der Länder Berlin und Brandenburg e. V. (2014). VERA 3 Vergleichsarbeiten in der Jahrgangsstufe 3 im Schuljahr 2013/14. Länderbericht Brandenburg. https:// www.isq-bb.de/wordpress/wp-content/uploads/2016/06/VERA_3_Bericht_2014_BB.pdf. Zugegriffen: 23. Nov. 2018.

KMK (2005). Bildungsstandards im Fach Deutsch für den Primarbereich (Jahrgangsstufe 4). Bonn: Kultusministerkonferenz.

Lanza, S. T., \& Cooper, B. R. (2016). Latent class analysis for developmental research. Child Development Perspectives, 10(1), 59-64.

Löffler, I., \& Meyer-Schepers, U. (2005). Orthographische Kompetenzen: Ergebnisse qualitativer Fehleranalysen, insbesondere bei schwachen Rechtschreibern. In W. Bos, E.-M. Lankes, M. Prenzel, K. Schwippert, R. Valtin \& G. Walther (Hrsg.), IGLU Vertiefende Analysen zu Leseverständnis, Rahmenbedingungen und Zusatzstudien (S. 81-108). Münster: Waxmann.

Löffler, I., \& Meyer-Schepers, U. (2009). Auswertung nach dem linguistischen Kompetenzmodell. In R. Valtin \& B. Hofmann (Hrsg.), Kompetenzmodelle der Orthographie. Empirische Befunde und förderdiagnostische Möglichkeiten (S. 60-74). Berlin: Deutsche Gesellschaft für Lesen und Schreiben.

Masyn, K.E. (2013). Latent class analysis and finite mixture modeling. In T. D. Little (Hrsg.), Oxford library of psychology. The Oxford handbook of quantitative methods: statistical analysis (S. 551-611). Oxford: University Press.

May, P. (1990). Kinder lernen rechtschreiben: Gemeinsamkeiten und Unterschiede guter und schwacher Lernen. In H. Brügelmann \& H. Balhorn (Hrsg.), Das Gehirn, sein Alfabet und andere Geschichten (S. 245-253). Konstanz: Faude.

May, P. (1993). Vom Umgang mit Komplexität beim Schreiben. Herausbildung orthographischer Kompetenz als erweiterte Rekonstruktion sprachlicher Strukturen. In H. Balhorn \& H. Brügelmann (Hrsg.), Bedeutungen erfinden - im Kopf, mit Schrift und miteinander (S. 277-289). Konstanz: Faude.

May, P. (2012). HSP 1-10 Hamburger Schreib-Probe. Manual/Handbuch: Diagnose orthographischer Kompetenz zur Erfassung der grundlegenden Rechtschreibkompetenzen. Stuttgart: Klett.

Moll, K., \& Landerl, K. (2010). SLRT-II. Lese- und Rechtschreibtest. Weiterentwicklung des Salzburger Lese-Rechtschreibtests (SLRT). Bern: Huber.

Mücke, S., \& Schründer-Lenzen, A. (2008). Zur Parallelität der Schulleistungsentwicklung von Jungen und Mädchen im Verlauf der Grundschule. In B. Rendtorff \& A. Prengel (Hrsg.), Kinder und ihr Geschlecht (S. 135-148). Opladen: Budrich.

Muthén, L. K., \& Muthén, B. (2012). Mplus user's guide. (7. Aufl.). Los Angeles: Muthén \& Muthén.

Nylund, K. L. (2007). Latent transition analysis: modeling extensions and an application to Peer victimization. A dissertation submitted in partial satisfaction of the requirements for the degree doctor of philosophy in education. www.statmodel.com/download/Nylund\%20dissertation\%20Updated1.pdf. Zugegriffen: 23. Juli 2020.

Ryoo, J. H., Wang, C., Swarer, S. M., Hull, M., \& Shi, D. (2018). Longitudinal model building using latent transition analysis: an example using school bullying data. Frontiers in Psychology, 9, 675. https:// doi.org/10.3389/fpsyg.2018.00675.

Scheerer-Neumann, G. (1989). Rechtschreibschwäche im Kontext der Entwicklung. In I. M. Naegele \& R. Valtin (Hrsg.), LRS in den Klassen 1-10 (S. 25-35). Weinheim: Beltz.

Scheerer-Neumann, G. (2015). Lese-Rechtschreib-Schwäche und Legasthenie. Grundlagen, Diagnostik und Förderung. Stuttgart: Kohlhammer.

Scheerer-Neumann, G., \& Schnitzler, C.D. (2009). Rechtschreiberwerb im zweiten Schuljahr. Zeitschrift für Grundschulforschung, 2, 95-110.

Schneider, W. (Hrsg.). (2008). Entwicklung von der frühen Kindheit bis zum frühen Erwachsenenalter. Weinheim: Beltz.

Schneider, W. (2017). Lesen und Schreiben lernen. Wie erobern Kinder die Schriftsprache? Berlin: Springer.

Schründer-Lenzen, A. (2013). Schriftspracherwerb (4. Aufl.). Wiesbaden: Springer.

Siekmann, K., \& Thomé, G. (2012). Der orthographische Fehler. Grundzüge der orthographischen Fehlerforschung und aktuellen Entwicklungen. Oldenburg: Isb. 
Spika, V. (2015). Die Kybernetische Methode im Schriftspracherwerb. Eine Wirksamkeitsstudie unter besonderer Berücksichtigung von Kindern mit ungünstiger Lernausgangslage. Augsburg: Universität Augsburg.

Spitta, G. (1988). Geben wir den Kindern Zeit, damit sie aus den Fehlern lernen können! Die Grundschulzeitschrift, 2(12), 6-12.

Spörer, N., Maaz, K., Vock, M., Schründer-Lenzen, A., Luka, T., Bosse, S., Jäntsch, C., \& Vogel, J. (2015). Wie entwickeln sich Kinder im inklusiven Unterricht? Zur Anlage der wissenschaftlichen Begleitung des Projekts „Inklusive Grundschule“. In D. Blömer, M. Lichtblau, A.-K. Jüttner, K. Koch, M. Krüger \& R. Werning (Hrsg.), Perspektiven auf inklusive Bildung. Gemeinsam anders lehren und lernen (S. 297-302). Springer VS: Wiesbaden.

Stranghöner, D., Hollmann, J., Otterpohl, N., Wild, E., Lütje-Klose, B., \& Schwinger, M. (2017). Inklusion versus Exklusion: Schulsetting und Lese-Rechtschreibentwicklung von Kindern mit Förderschwerpunkt Lernen. Zeitschrift für Pädagogische Psychologie, 31(2), 125-136.

Thomé, G. (2003). Entwicklung der basalen Rechtschreibkenntnisse. In U. Bredel, H. Günther, P. Klotz, J. Ossner \& G. Siebert-Ott (Hrsg.), Didaktik der deutschen Sprache (S. 369-379). Paderborn: Ferdinand Schöningh.

Thomé, G., \& Gomolka, J. (2007). Rechtschreiben. In B. Beck \& E. Klieme (Hrsg.), Sprachliche Kompetenzen. Konzepte und Messung. DESI-Studie (S. 140-146). Weinheim: Beltz.

Valtin, R. (1988). Schriftspracherwerb als Entwicklungsprozeß. Grundschule, 20(12), 12-16.

Valtin, R., \& Sasse, A. (2018). Angemessen fördern - mithilfe des Stufenmodells. Grundschule, 6, 12-15.

Weinert, F.E., \& Helmke, A. (1997). Entwicklung im Grundschulalter. Weinheim: Psychologie Verlagsunion. 